\title{
LA OBJETIVIDAD DE LOS NÚMEROS FREGEANOS
}

ALBERTO MORETTI

Universidad de Buenos Aires

Conicet

Al menos una parte de lo que sostiene el realismo acerca de la aritmética, consiste en la tesis de que las afirmaciones aritméticas son verdaderas o falsas, hablan acerca de objetos y la existencia de estos objetos así como el valor veritativo de esas oraciones son independientes de las capacidades cognoscitivas de los seres humanos. Desde el punto de vista tradicional el valor veritativo de una oración se funda en las propiedades de los objetos de los que la oración habla. Así, la cuestión ontológica básica se asienta en la naturaleza de esos objetos, resumiéndose - habitualmente - en la pregunta de si esas entidades son acontecimientos o productos mentales o existen independientemente de la actividad mental. Llamaré $R$ a la tesis realista así entendida.

Cuando la discusión tradicional entre realistas y opositores se plantea de modo semántico aparece, prima facie, como un asunto sobre la naturaleza de las entidades referidas por los términos singulares (y generales). M. Dummett, sin embargo, ha considerado que éste no es el modo correcto de exponer el problema. En su opinión, la disputa sólo tiene genuino sentido si es vista como si se refiriera al modo de atribuir significados a las oraciones. ${ }^{1}$ Según su enfoque, si un hablante puede

${ }^{1}$ Cfr. "What is a Theory of Meaning? (II)", en Evans y McDowell 
aseverar una oración $\alpha$ entonces puede captar el contenido o significado de $\alpha$, esto es, si usa correctamente $\alpha$ (si posee, en algún sentido, las condiciones de aseveración de $\alpha$ ) entonces capta su significado. Además, captar el significado de $\alpha$ no puede ser más que lo que cabe advertir conductualmente, es decir, si el sujeto aprehendio el significado de $\alpha$ entonces adquirio la capacidad de aseverar $\alpha$ cuando es correcto hacerlo (sabe usar $\alpha$ ) y en esto se agota el conocimiento que constituye conocer el significado de $\alpha$. Cualquier otro componente del significado que fuera ajeno a toda diferencia conductual sería irrelevante para la comprensión (interpretación). Y toda teoría del significado debe serlo de la comprensión. Las condiciones de aseveración, por su lado, dependen de las condiciones de aseveración y falsificación de $\alpha$, más precisamente, de la capacidad del sujeto para reconocer esas condiciones --que constituyen su fundamento para la aseveración-cuando existen. Los significados oracionales quedan, por tanto, esencialmente ligados a los usos públicos y a la importancia cognoscitiva de esos usos, y la idea de falsificación o verificación resulta la clave del análisis semántico. ${ }^{2}$ Aplicando este enfoque el problema del realismo se traslada desde la caracterización de los referentes de los términos a la determinación del significado de las oraciones: de la ontología a la semántica y la gnoseología. ¿Qué razones justifican el desplazamiento?

La objeción dummettiana contra el realismo tradicional ejemplificado en $R$, cobra la forma de un dilema: o bien $R$ es ininteligible o metafórica, pero en todo caso teóricamente vacua,

(comps.), Truth and Meaning, Clarendon, Oxford, 1976; Elements of Intuitionism, Clarendon, Oxford, 1977, especialmente cap. 7; Truch and Other Enigmas, Duckworth, Londres, 1978, especialmente prefacio y ensayos 1, 14 y 21 .

2 Algunos rasgos conexos son: (i) la prioridad (fregeana) de las oraciones sobre las expresiones suboracionales; (ii) el rechazo del holismo en favor del "molecularismo" (oracional) semántico, rechazo derivado de la conjunción entre el papel clave otorgado a la idea de asertabilidad y el requisito de hacer posible la explicación de los cambios de significado. 
o bien $R$ es un modo equívoco de expresar que el significado de las oraciones aritméticas depende de sus condiciones veritativas y éstas son independientes de las capacidades humanas para reconocerlas. ${ }^{3}$ La segunda opción se resume frecuentemente en la tesis de la validez del principio de la bivalencia para las oraciones aritméticas. Discutir sobre la objetividad de los números es entonces una tarea superflua, la cuestión fundamental es la de la objetividad de las oraciones aritméticas y su solución no presupone sino que, por el contrario, sugiere una solución de aquella disputa a quien siga concediéndole algún interés adicional. Y, según se desprende de las ideas semánticas esbozadas en el párrafo anterior, $R$ es falsa: no puede darse sentido a una noción de verdad que presuponga (o al menos admita) la posiblidad de condiciones verificatorias o falsatorias inalcanzables por los seres humanos y que, a la vez, pretenda explicar el significado oracional.

$\mathrm{Si} R$, explícita y básicamente, sólo predica sobre entidades referidas, ¿por qué debería construirse como si hablara fundamentalmente del significado de oraciones y de la idea de verdad? A este respecto Dummett ha ofrecido algún barrunto de argumentación directa y, además, su objeción principal contra el realismo presenta una vía indirecta de respuesta.

Siguiendo a Frege, Dummett mantiene que el significado y también la referencia de las partes (partes según alguna gramática) de las oraciones —en particular los términos singulareses función del significado o la referencia, según corresponda, de las oraciones mismas. Esto quiere decir, o bien que el teórico o el hablante del lenguaje sólo pueden atribuir referencia a las partes sobre la base del significado que hayan atribuido a la oración, o bien que los términos sólo tienen referencia cuando

3 Sostener - como hace el realista dummettiano-que las condiciones veritativas de las oraciones son independientes de las capacidades cognitivas humanas no implica afirmar que de hecho haya condiciones veritativas para las que no pueda concebirse manera (¿humana?) alguna de reconocerlas, sólo es compatible con eso. 
son usados en oraciones. Pero en cualquier caso este principio supone que las partes tienen referencia propia (cuando la tienen, aun si eso sólo ocurre por y al ser usadas en oraciones, les es propia), esto es, su referencia es derivada pero es específica. Sin embargo, de aquí Dummett concluye que "toda tesis concerniente al status ontológico de los objetos de una clase dada debe ser, al mismo tiempo, una tesis sobre lo que hace verdadero a un enunciado que involucre la referencia a tales objetos, en otras palabras, una tesis acerca de qué propiedades puede tener un objeto de esa clase". 4 No obstante, que un $\alpha$ deba ser un $\beta$ no dice por sí mismo que el primero sea idéntico o mejor, que se reduzca al $\beta$, ni menos que — tratándose de una oración- no se entienda como $\alpha$ sino como $\beta$. Esa correlación no es obstáculo para otorgarle un sentido legítimo a las afirmaciones sobre la naturaleza de ciertos objetos, que sea diferente del que les corresponda a sus correlativas afirmaciones sobre condiciones veritativas. Por otro lado, si es plausible asimilar las tesis sobre status de objetos a tesis sobre las propiedades que tienen o las clases a que pertenecen esas entidades, no lo es _pace Dummett_- asimilar estas últimas a tesis sobre condiciones veritativas. A menos, claro está, que se presuponga lo que debería demostrarse: que la estructura profunda del problema del realismo es de tipo semántico.

Un segundo argumento discurre en torno de la fundamentación de la divergencia que en materia de lógica separa a intuicionistas y platónicos. Dummett se esfuerza por mostrar que ninguna toma de partido en la disputa ontológica es capaz de dirimir la cuestión lógica, excepto bajo las suposiciones demasiado restrictivas del finitismo estricto, en tanto que este problema tiene respuesta luego de zanjar la cuestión semántica presuntamente subyacente a la ontología. ${ }^{5}$ Se advierte que esta

4 Truth and Other Enigmas, p. 230.

5 Llegado aqur Dummett sostiene que, siendo intuicionismo y platonismo claros ejemplos de antirrealismo.y realismo, y como la discusión en- 
defensa del enfoque semántico de la ontología se basa en su fertilidad para resolver un problema de lógica. ${ }^{6}$ Pero no puede desatenderse el hecho crucial de que ese problema ya es un asunto relativo a oraciones (esto es, a tesis lógicas conjeturales). Y no es extraño que, al concernir al significado de expresiones no referenciales —básicamente, signos lógicos- su solución dependa de los modos de atribución de significado oracional.

Un resumen de los motivos de Dummelt en contra del realismo, ahora tal como él propone entenderlo, es el siguiente. La primera premisa es un versión de la teoría que hace del significado una función del uso y de las capacidades epistémicas humanas. La segunda afirma que existen oraciones que tienen claras condiciones de asertabilidad, esto es, se sabe en qué consiste una garantía para aseverarlas y podría ser reconocida si se diese, pero son oraciones para las cuales no existe modo alguno de alcanzar esa garantía, son indecidibles. Por consiguiente, la noción de verdad que corresponde ligar con la noción de significado impide la validez irrestricta del principio de bivalencia, i.e., implica el no realismo. De donde, si alguien creyese aún que la naturaleza de los objetos referidos explica el valor veritativo de las oraciones, se vería conducido a creerla dependiente de las capacidades cognitivas humanas. De este modo vuelve a confirmarse la hipótesis del carácter esencialmente semántico (y relativo a oraciones) de la tesis realista.

Era previsible que ambas premisas merezcan reparos. Respecto de la primera, junto con la observación de que es muy

tre ellos resultó semántica y general (i.e. no específicamente matemática), entonces todo caso de controversia acerca del realismo ha de ser de igual índole. Para un hombre que desespera de las analogías y las extrapolaciones, esta inferencia debería haber traído prudente desasosiego; no lo trajo.

6 No un problema menor, es cierto. Sobre todo para quienes centran las discusiones de filosofía de la matemática en la aceptabilidad de los métodos de demostración utilizados. Ésa es una decisión programática atractiva, pero no única. 
dudoso que la teoría de las condiciones de asertabilidad logre explicar el significado-uso de todas las construcciones oracionales cognitivamente importantes, ${ }^{7}$ debe notarse que Dummett no ha probado que no exista modo alguno de caracterizar las condiciones de asertabilidad de las oraciones que sea compatible con la validez general del principio de bivalencia. Con relación a la segunda poco puede decirse hasta la aclaración de asuntos decisivos como la indecidibilidad: ¿para quién y según qué tipo de restricciones a la noción de método decisorio? Si la idea de decidibilidad fuese la vinculada con la construcción de pruebas en los sistemas axiomáticos, entonces una oración indecidible lo sería siempre en relación con algún sistema, no en absoluto. Para cada oración indecidible $\alpha$ existirá un sistema en que deje de serlo: aquel que incluye $\alpha$ o su negación entre los axiomas. La cuestión, claro, estará en los motivos para adoptar axiomas y sobre esto los realistas tendrán razones programáticas y también de fertilidad teórica para justificar sus elecciones. ${ }^{8}$ Por otro lado, mantener no ya la existencia sino la mera posibilidad de que haya oraciones indecidibles es ya la negación del realismo, que no es una tesis extensionalista - aunque suela formularse así - sino una afirmación metafísica y por eso pretendidamente necesaria. Y un argumento contra el realismo que tiene una premisa equivalente a su negación no es un argumento eficaz. Tal vez la premisa se elabore del siguiente modo: en el ámbito epistémico (humano) es razonable creer en la posibilidad de la existencia de oraciones indecidibles, esto es, creer que la bivalencia epistémica es ilegítima. En este caso onus probandi es de quien la defienda. Entonces - se dirá luego- si este principio no se justifica como medio para adquirir conocimiento, ¿́por qué

7 Cfr. por ejemplo, D. Edgington, "Do Conditionals Have Truth-Conditions?", Crítica, vol. XVIII, no. 52, abril de 1986.

B Cfr. por ejemplo, K. Gödel, "What is Cantor's Continuum Problem?", en Benacerraf y Putnam (comps.), Philosophy of Mathematics, PrenticeHall, 1964. 
razón podría sostenerse su validez en el mundo? Pero este modo de plantear la cuestión conduce otra vez al problema general de la relación entre gnoseología y ontología. Y en este terreno ambos partidos (realistas y no realistas) deben producir tesis argumentadas.

Este bosquejo, sin duda, no hace justicia a los detalles de la discusión, pero al menos destaca algunos de los principales puntos a tener en cuenta y sugiere que las respuestas no son claras. Como consecuencia — se espera-quedará suficientemente expedita la vía para considerar seriamente la pregunta de la objetividad de los números.

Según parece la aritmética es un discurso informativo donde se habla de números. Frege pensó que la apariencia está justificada. Según él, en efecto, las afirmaciones numéricas resultan ser aseveraciones acerca de conceptos, en rigor, asignaciones de números a conceptos. ${ }^{9}$ También es cierto que los teoremas aritméticos van acompañados de un fuerte sentimiento de certeza. Frege ofrecio una explicación de este sentimiento mediante la tesis —que compartía con Kant-de objetividad, i.e., la no subjetividad de la aritmética; tesis que él - a diferencia de Kant- desarrolló en términos de análisis conceptuales y no de construcciones en la intuición pura. Ambos esfuerzos conducían a una atribución de necesidad o por lo menos de independencia respecto de la subjetividad empírica, que justifica la presencia de sentimientos de certeza y permite la posiblidad de su ausencia sin desmedro del valor cognoscitivo de las aserciones.

Desde la perspectiva de Frege las afirmaciones aritméticas básicas vinculadas con la operación de contar, tales como 'El número de gatos verdes es igual a uno' son, bien entendidas;

${ }^{9}$ Die Grundlagen der Arithmetik, Breslau, 1884 (en adelante $G L$ ), $\$ 45$, $46,52,57$. 
asignaciones de números a conceptos. En el caso dado tenemos: 'El número correspondiente al concepto de gato verde es el uno' o, mejor, 'El concepto de gato verde tiene al uno como número correpondiente'. La oración 'El duque de Alba tiene al cardenal Antúnez como invitado ilustre' exhibe aparentemente la misma forma que la anterior; sin embargo, Frege no admitía que un término singular pudiese referirse a un concepto, por lo cual las transformaciones deben continuar generando, primero algo como 'Hay un objeto que es un gato verde y el concepto de ser gato verde pero distinto de ese objeto tiene al cero como número correspondiente', hasta obtener quizás 'Gato verde sólo hay uno' donde la luz semántica se ha hecho.

Hay, entonces, asignaciones de números a conceptos. Pero los conceptos fregeanos no son entidades mentales en modo alguno. No son objetos, pero en el sentido de que no tienen todas las características metafísicas de los objetos, no en el sentido de que sean creaciones o aspectos de cada sujeto particular. Dicho pintorescamente: no son objetos pero son objetivos (i.e. no son subjetivos). ${ }^{10}$ Dado esto, si además ocurriese, o bien que los numerales fuesen eliminables sin pérdida de conocimiento, o bien que los números también fuesen - como los conceptos - independientes de las capacidades epistémicolingüísticas de los hombres, quedaría asegurada la objetividad de las afirmaciones aritméticas (excluyendo la posibilidad de vacuidad referencial).

Frege considera una vía hacia la eliminabilidad ${ }^{11}$ basándose en las transformaciones ejemplificadas más arriba donde los numerales desaparecen en favor de los cuantificadores. No obstante, la explicación del número y las afirmaciones numéricas deben fundamentar todos los contextos "útiles para la ciencia" ${ }^{2} \mathrm{y}$, según su punto de vista, la vía anterior no permite en-

$\begin{array}{ll}10 & G L, \S 47 . \\ 11 & G L, \S 55 . \\ { }^{12} & G L, \S 57 .\end{array}$ 
tender adecuadamente afirmaciones que son teoremas típicos como ' $1+1=2$ ' o informaciones de conteos como 'El número de gatos verdes es el mismo que el de perros verdes', ni decidir sobre la verdad o falsedad de casos como 'El número correspondiente al concepto de abogado es Julio César' o 'Julio César es un número'. ${ }^{13}$ Por extrañas que suenen, Frege está obligado a admitir oraciones como las anteriores, ya que su predicado de identidad está definido para todo objeto. Es intuitivo fundar el rechazo de estas afirmaciones en las características conocidas de Julio César, esto es, en características que los números no deberían tener (al menos en su totalidad). Esto promueve la búsqueda de alguna caracterización positiva de los números. La mejor respuesta a esta inquietud se consigue tratando de encontrar definiciones explícitas de los números y del número en general. Frege se ve llevado entonces, en primer lugar, a conjeturar que los números son objetos. Ofrece algunos motivos sintáctico-semánticos para abonar su posición ${ }^{14}$ y aduce, además, sobre la base de sus tesis ontológicas, que si los números no fuesen objetos serían conceptos y hay razones para negar que sean conceptos. ${ }^{15}$ Más tarde destacaría también que los números mismos son contables, cosa incomprensible si no se tratase de objetos. ${ }^{16}$ En segundo lugar, le parece claro que los números no pueden ser objetos internos ${ }^{17}$ ni externos al modo del mundo físico. ${ }^{18}$ Esto es, de ellos no tenemos intuición alguna; no son dados ni al sentido interno ni al externo.

En estas circunstancias se presenta una alternativa interesante. $\mathrm{O}$ bien los números son objetos de existencia independiente de la mente, aprehensibles sólo a través de represen-

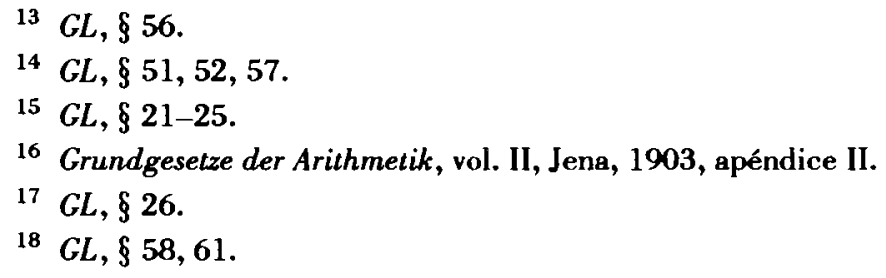


taciones conceptuales; o bien son objetos constituidos(bles) y simultáneamente aprendidos(bles) por la operación meramente conceptual de la mente, es decir, no resultado de síntesis de intuiciones sino de análisis de conceptos como los cuantificadores, $o$, tal vez más precisamente, de análisis de juicios posibles o de conceptos de juicios posibles. En ambos casos se asegura la objetividad de la aritmética convirtiéndola en una teoría analítica acerca de objetos puramente lógicos, mostrando así que es una teoría acerca de todos los objetos pensables, no solamente de los empíricamente cognoscibles en sentido kantiano, ${ }^{19}$ con lo cual la idea de número queda conceptualmente ligada con la noción general de entidad u objeto. Debe notarse que la preocupación fregeana central es que si lo que es verdadero de los números puede o no variar respecto de cada suje o del que pueda decirse que piensa. Resulta entonces más básico dar respuesta a la cuestión de si esos entes son "los mismos para todos" o son del tipo de las imágenes psíquicas individuales, que resolver si las entidades referidas por los numerales son extramentales o no. Aquí reside el núcleo del problema de la objetividad de la aritmética. La primera opción de la alternativa descrita al comienzo es una respuesta drástica a la pregunta fundamental, pero la segunda opción también es una respuesta afirmativa viable. Al menos mientras el concepto de construcción mental involucrado permanezca en el estadio de imprecisión con que habitualmente aparece en estas discusiones.

Frege no discute ni propone la segunda opción, adopta la primera y pasa a explicar de qué manera podemos representarnos conceptualmente estos objetos autosubsistentes: los números. Combina la idea platónica ${ }^{20}$ del carácter fundamental de las relaciones de identidad y diferencia en el ámbito de los objetos (idea que en el terreno gnoseológico conduce a poner

$$
\begin{aligned}
& G L, \oint 14 . \\
& C f r . \text { Sofista, } 254 \mathrm{a} .
\end{aligned}
$$


énfasis en la formulación de criterios de identificación de objetos) con su principio metodológico - derivado también de tesis ontológicas - que prescribe que para buscar la referencia de los términos se examinen sus usos oracionales básicos. A partir de aquí produce su célebre análisis de las afirmaciones de identidad numérica.

Lo dado es lo expresado en un juicio. Lo involucrado en un juicio son objetos y conceptos. La objetividad de un juicio, esto es, aquello que justifica su asertabilidad terminará siendo, sorprendentemente, la referencia al objeto verdad. Pero esta referencia, para el caso de las afirmaciones aritméticas, ha de producirse mediante la predicación de conceptos numéricos. Si los números fuesen objetos del tipo para cuya representación se requieren intuiciones empíricas, entonces la aritmética sería contingente. De modo que a fin de asegurar la necesidad de las afirmaciones aritméticas cuando se quiera explicar la posibilidad de representación de los números, podrán considerarse caminos como postular una intuición de objetos ideales o una intuición pura constituyente o una captación no intuitiva resultante del actuar puramente conceptual, o simplemente negar que el sujeto capte los números en modo específico alguno (si algo puede "captar" de ellos es sólo que existen) para sostener que la relación que los vincula es la de postulación, la que, a su vez, pude alcanzar cierta justificación indirecta a través de la utilidad de la aritmética para el conocimiento empírico. La vía fregeana no iguala objetividad con intersubjetividad (sea respecto de alguna comunidad ideal de sujetos epistémicos o respecto de algún conjunto de capacidades epistémicas más o menos caracterizable), ni la hace corolario de alguna subjetividad trascendental, sino que la entiende como independencia de toda subjetividad y eso excluye también las justificaciones pragmáticas.

Por otra parte, los números de Frege son objetos autosubsistentes de una naturaleza tal que no llegan a la conciencia mediante intuiciones singulares. Su "naturaleza" entonces se 
ha de indagar estudiando las aserciones elementales donde se hace referencia a ellos, esto es, aquellas donde se identifican los números asociados con diferentes conceptos. $\mathrm{El}$ análisis fregeano comienza estableciendo una condición cuyo cumplimiento se requerirá para aceptar cualquier propuesta de aclaración de la idea de número. Frege exige que pueda probarse que la equinumerosidad de las extensiones de los conceptos $F$ y $G$ es condición materialmente necesaria y suficiente de la igualdad de los números asociados con $F$ y $G$. El hecho de que la equinumerosidad sea una relación de equivalencia le permite proponer su famosa definición: el número de un concepto $F$ es la extensión del concepto equinumerable con $F$. Y un número no es otra cosa que el número de algún concepto. Finalmente puede probar que de esta definición se deduce el requisito de aceptabilidad impuesto y que con ella se satisfacen las leyes aritméticas.

Husserl formuló dos objeciones al "intento" de Frege: (i) el carácter extensional de la definición fregeana la hace insuficiente como aclaración de la idea de número; para lograr ese objetivo, una definición debería hacer explícito el contenido del concepto de número y no meramente su extensión; ${ }^{21}$ (ii) la noción de número es primitiva, por ende el único modo de fundamentarla es remitiéndose a la actividad mental que ocasiona su aparición; de un concepto básico no puede darse definición. ${ }^{22}$ La primera objeción no varió en su obra posterior; la segunda también se mantuvo pero cambió su tono empírico del periodo psicologista por un esforzado trascendentalismo en la etapa fenomenológica.

21 Philasophie der Aritmethik, La Haya, 1891 en Husserliana, La Haya, 1970 , t. XII, cap. VII, in fine.

22 Ibid., cap. VII, "Freges Versuch" (antes en "Über den Begriff der Zahl: Psychologische Analysen", 1887), introducción, in fine. 
Desde el punto de vista de Frege la primera crítica se supera teniendo en cuenta dos cosas. En primer lugar, que el contenido cognoscitivamente importante de una expresión conceptual es su referencia, no su componente intensional, pero esta referencia no es una extensión sino una función. La definición del concepto ser (el) número de $F$, no consiste en explicitar su extensión sino en afirmar que ser (el) número de $F$ es ser la extensión del concepto ser equinumerable con $F$. La clase de las propiedades equinumerables con $F$ no es la extensión del concepto de número $F$ sino la del concepto equinumerable con $F$. De forma correspondiente, ser un número es estar, con un concepto $F$, en la relación de ser idéntico con la extensión del concepto de ser equinumerable con $F$. En segundo lugar, que no es posible definir un concepto ofreciendo otro del que pueda decirse que tiene idéntico contenido. Lo impide el hecho de que la relación de identidad sólo tiene aplicación entre objetos; la relación entre conceptos que desempeña un papel análogo a la identidad entre objetos es, precisamente, la relación de tener idéntica extensión. Es cierto entonces que una definición del concepto de ser número de $F$ en términos del concepto de ser la clase de los conceptos equinumerosos respecto de $F$, sólo muestra dos conceptos de los que afirma que tienen igual extensión, pero esto es todo lo que es posible hacer. ${ }^{23}$

La segunda objeción es resultado de lo que para Husserl debe ser la estrategia correcta para la comprensión de un concepto primitivo: el análisis psicológico de su contenido. Y su manera de llevarlo a cabo reposa en una larga tradición, iniciada por Aristóteles, que atribuye a las mentes humanas la capacidad de efectuar ciertos procesos (las abstracciones) generadores de representaciones mentales generalizadoras (los conceptos). En el comienzo están las multiplicidades concre-

23 "Rezension von: E.G. Husserl, Philosophie der Aritmethik I", 1894 en Kleine Schriften, Darmstadt, 1967, pp. 318 ss. 
tas, que habrán surgido mediante un acto mental unificador de las intuiciones de múltiples objetos. La operación abstractiva pone entré paréntesis las propiedades individualizadoras de los objetos reunidos y los trasforma en "algos" que aún permanecen reunidos, originando así una multiplicidad abstracta, es decir, un número.

Que la estructura lógica de un concepto deba estudiarse psicológicamente $^{24}$ es una proposición que exasperaba a Frege. Su trabajo abunda en razones para rechazar este enfoque, razones de índole general y también especiales respecto de la eficacia constructiva de la abstracción. Una variación sobre la crítica de Husserl puede iniciarse distinguiendo entre un sentido ontológico y otro gnoseológico de la idea de fundamentación. Según el primero se buscarán, por ejemplo, jerarquías y relaciones entre tipos de entidades y procesos dando lugar a diferentes conceptos y tramas conceptuales. De acuerdo con el otro, se intentará descubrir cómo es posible que los sujetos epistémicos tengan acceso a las entidades o procesos que existen, p:eguntando entonces por la legitimidad epistémica de los concestos correspondientes. Sólo falta agregar ahora que de las nociones ontológicamente elementales no cabe otra fundamentación que la gnoseológica. ${ }^{25} \mathrm{Si}$ la de número es una de éstas, entonces, si no se explica satisfactoriamente cómo se accede epistémicamente a los números, no se ha justificado la validez objetiva de la noción de número. La negación del carácter básico del concepto de número es, desde luego, una de las tesis principales que Frege pretende demostrar, pero su fracaso y lo turbio de la noción de conjunto que vino a continuar el intento, renuevan la posición de Husserl. Estas observaciones se em-

24 Esta exigencia, y en estos términos, se encuentra en la tesis de habilitación de 1887 ya citada, cfr. pp. $46-47$.

25 Sobre la imposibilidad de dar fundamentos lógicos de los conceptos primitivos, cfr. Frege, Grundsetze, vol. I, Jena, 1893, p. xvii. 
parientan con las que más recientemente hiciera Benacerraf ${ }^{26}$ y se vinculan con la difícil cuestión general de las relaciones que sea sensato alentar entre las atractivas tesis ontológicas y las generalmente amargas reflexiones gnoseologicas. Sobre esto algo se dijo, en vena fregeana, en la sección anterior. Un punto clave está en la justificación de las restricciones que se impongan a la idea de relación epistémica, por ejemplo: ¿es necesario que incluya nexos causales?, si lo fuera, ¿cómo entender, ontológicamente, la causalidad?

También puede procurarse una revaloración de las objeciones de Husserl y de su estrategia teórica sobre la base de que la definicion fregeana es arbitraria debido a que una infinitud de otros objetos cumplen las condiciones de adecuación impuestas y Frege no da motivos para compartir su elección. ${ }^{27}$ El recurso al método abstractivo aparece entonces como un modo de justificar cl que se haya adoptado cierta tesis sobre la naturaleza de los números $\mathrm{y}$, al mismo tiempo, proveería de una respuesta más o menos clara al interrogante sobre la posibilidad de su conocimiento. El problema aquí es formular el método de manera que escape a la convincente crítica que Frege dirigiera en su contra. En Peano y luego más claramente en Weyl y Lorenzen se ha querido ver un esfuerzo exitoso en esta dirección. ${ }^{28} \mathrm{La}$ tradición aristotélica procede mediante la desatención creciente a las propiedades de un objeto a fin de producir otro objeto - abstracto- constituido por un subconjunto de las propiedades de la entidad dada a la intuición —entidad concreta. La nueva versión se traslada al campo lingüístico. Desde un conjunto de objetos concretos pasa al con-

${ }^{26}$ P. Benacerraf, "Mathematical Truth", J. of Ph., vol. LXX, no. 19, noviembre, 1973.

27 Benacerraf expone algo similar en otro famoso artículo: "What Numbers Could not Be", Phil. Rev., vol. LXXIV, enero, 1965.

28 Cfr. I. Angelelli, "Abstraction, Looking-Around and Semantics", Studia Leibnitiana, cuaderno 8, 1979; y "Husserl-Frege: Filosofía del número", Análisis filosófico, vol. IX, no. 2, noviembre, 1989. 
junto de los predicados presentes en un conjunto elegido de oraciones consideradas verdaderas acerca de esos objetos. Se define luego una relación de equivalencia entre los objetos dados que permite dar el paso abstractivo que ahora consiste en prescindir de todo predicado que no sea invariante respecto de la relación de equivalencia. De este modo muchos objetos concretos diferentes se hacen indiscernibles dentro del nuevo lenguaje. Se pretenderá entonces que se ha producido un objeto nuevo, abstracto, constituido sólo por las propiedades asociadas con los predicados invariantes. Donde la tradición ve un acto de desatención de propiedades de cosas, un acto de limitación intuitiva, los presuntos herederos ven la definición de una equivalencia entre cosas - un acto que escapa a la mera intuición de objetos-y un acto de limitación lingüística.

Se buscaba evitar arbitrariedades pero, ¿cómo se justifica la elección del conjunto inicial de oraciones verdaderas que suministrarán los predicados cruciales?, ¿̇y cómo la de la equivalencia que se habrá elegido entre muchas? Recuérdese también que ambas elecciones dependerán del conjuto de objetos dados inicialmente, con lo que es posible que las propiedades que emerjan para constituir el objeto abstracto difieran según cuáles sean, en cada caso, esos objetos. Cuando Frege "construye" un conjunto de propiedades, los nuevos abstraccionistas "construyen" un objeto parcialmente determinado por sólo las propiedades de ese conjunto. En ambos casos el peso de la construcción recae en la equivalencia elegida. En ambos casos esta elección tiene el mismo grado de arbitrariedad. ¿Son mayores las diferencias con el método de Frege que las diferencias con la tradición aristotélica? En lo que importaba, la supuesta arbitrariedad, la respuesta es negativa.

Por otra parte, tanto la abstracción moderna como el método fregeano pueden verse de dos modos. La abstracción como forma de acceso epistémico a ciertas entidades o como método para crear entidades. El análisis de Frege como un modo de generación de hipótesis acerca de entidades o como método pu- 
ramente conceplual para dar existencia a entidades. A su vez, cuando los objetos en cuestión se considerasen construidos, si además se los tomase por efectivos, serían mentales, pero nada impide, prima facie, entenderlos como objetos meramente posibles (o, al menos, pensables) y de naturaleza (aunque no origen) extramental. En este último caso, la creencia de Frege en la no creatividad de las definiciones debería limitarse a la no creatividad de objetos efectivos.

La nueva abstracción no aparece, pues, como clara alternativa del método de Frege, superadora de sus presuntas limitaciones epistémicas y arbitrariedades ontologicas. Y, por otra parte, el análisis fregeano puede defenderse como un proceso, independiente de la intuición, que, o bien permite la creación de objetos puramente lógicos (dependientes de ciertos cuantificadores), obviando de este modo los cargos anteriores, o bien permite captar objetos meramente posibles, respecto de los cuales no cabría esperar acceso epistémico que involucrase relaciones histórico-causales y que, en tanto limitados al ámbito de lo pensable, no merecerían el calificativo de arbitrarios.

Algunas personas, de ascendencia kantiana, encontrarán embarazosa una idea de construcción de objetos que no otorgue un papel a las intuiciones, pero èpor qué esa idea debería implicar la de intuición pura o empírica? Quizá porque entonces parecería más sencillo explicar la aplicabilidad exitosa de la aritmética a la ciencia natural. Pero es obvio que esta ciencia recurre tanto a conceptos como a intuiciones; de modo que concebir la aritmética como una construcción puramente conceptual no le impide participar de sus variadas ilusiones. Cómo es más adecuado considerar la teoría fregeana, constructiva o platónicamente, dependerá del tipo de teorías de la mente, del mundo y de las posibilidades con las que se espera integrar sus resultados. ${ }^{29}$

29 Agradezco las observaciones de D. Edgington y R. Orayen, que me permitieron mejorar la versión original de este trabajo. También agradezco el apoyo que la Fundación Antorchas me brindara durante su preparación. 
In section 1 I discuss some arguments suggested by Dummett's work, aimed to support the claim that the real problem between realism and antirealism is one about the meaningfulness of sentences. I give some reasons to consider them less than conclusive, and to mantain the importance of the traditional approach in terms of the nature of the referred objects.

In section 2 I give an account of Frege's view on the objectivity of arithmetic and its relationship with the objectivity of numbers. I defend the possibility of a partially constructivistic interpretation of Frege's analysis.

In section 3 Husserl's criticisms of Frege are summed up. I claim that they are not efficient in their original version but it is also suggested that the objections can be rephrased in such a way that they come closer to Benacerraf's criticism on Platonism. An abstractionist alternative, connected with the Erlangen Program, is examined. According to what was said in section 2 , it is suggested that even though Frege's view is still unsatisfactory, it is not refuted and the abstractionist view is even weaker.

[Traducción de Raúl Orayen] 